\title{
Welch Based Denoising Technique for a Set of Chirp Signals Corrupted by Gaussian Noises
}

\author{
Fendy Santoso \\ Department of Electrical and Computer Systems Engineering \\ Monash University, VIC, 3800 \\ Melbourne, Australia \\ fendy.santoso@gmail.com
}

\begin{abstract}
The aim of this research is to investigate the performance of Welch based de-noising technique for a set of chirp signals corrupted by Gaussian noises. In telecommunications, chirp signals are widely studied, particularly for sonar, radar and spread spectrum applications. However, unlike conventional signals, chirp signals are typical of time varying frequency signals. It sweeps linearly from a low to a high frequency. It is in fact a signal in which its frequency increases or decreases with time. Results indicate that Welch based de-noising technique has effectively inhibited the noise. Nevertheless, this method works satisfactory only below its threshold point. Beyond this limit, the signal-to-noise ratio of the desired signal is not acceptable. Figuratively, radar can only detect the presence of the aircraft only at a certain limited distance only. As soon as the aircraft moves further apart, the transmitted signal becomes substantially weaker before the noise can completely overwhelm it. It turns out that the presence of the aircraft is no longer perceptible.
\end{abstract}

Keywords: Chirp Signals; Gaussian Noises; Welch Based Denoising Technique.

\section{Introduction}

Welch's method, named after P.D. Welch, is a technique applied to compute power spectra density (not the spectrum) of the signal. It is based on Barlett's procedure which splits a set of data into smaller sets of data and calculates the modified periodogram (the power spectrum) of each set. At the end, it comes up with an array of power measurements against its frequency "bin" [1].

The modified periodogram is calculated by applying a window function to the time-domain data, computing the discrete Fourier transform, and then computing the squared magnitude of the result.

Most window functions afford more influence to the data at the centre of the set than to data at the edges, which represents a loss of information. To mitigate that loss, the individual data sets are commonly overlapped in time.

Received April $8^{\text {th }}, 2008$, Revised November $29^{\text {th }}, 2008$, Accepted for publication November $29^{\text {th }}, 2008$. 
The individual periodograms are then time-averaged, which reduces the variance of the individual power measurements. For further details, interested readers may refer to [1], [2] and [3].

The organisation of this paper is as follows. Firstly, Section I discusses some background information related to the importance of this research and also the organisation of this paper. Section II deals with the underlying principles of chirp signals. Meanwhile, Section III looks at the simulation and analysis of the P-Welch denoising process upon a set of chirp signals. Conclusions are accordingly drawn in Section IV.

\section{Chirp Signals}

The well-known signals used in this research are called chirp signals. Unlike conventional signals, a chirp signal is a time varying frequency one. It sweeps linearly from a low to a high frequency. It is a signal in which its frequency increases or decreases with time. It is widely studied, particularly for applications in sonar and radar and also in spread spectrum communication technique. Interested readers are suggested to refer to $\{[4]-[9]\}$ for further details.

\section{System's Model, Assumptions and Simulations}

The chirp signal of our interest is linearly described in time domain as follows:

$$
x(t)=\left\{\begin{array}{ll}
\cos [\phi(t)] ; & t \in[1,12]] \\
0 & \text { elsewhere }
\end{array} .\right.
$$

The angle function $\varphi(t)$ is such that the frequency $f(t)$ increases linearly with time:

$$
f(t)=t+4 \text {. }
$$

Also, the instantaneous frequency is defined as derivative of $\varphi(t)$ as follows:

$$
f(t)=\frac{1}{2 \pi} \frac{d}{d t} \varphi(t)
$$

By substituting (2) into (3), we finally obtain:

$$
\varphi(t)=\left(\pi t^{2}+4 \pi t\right),
$$

Accordingly we obtain, 


$$
x(t)=\left\{\begin{array}{l}
\left.\cos \left[\pi t^{2}+4 \pi t\right] ; \quad t[1,12]\right] \\
0 \quad \text { elsewhere }
\end{array} .\right.
$$

The distribution of chirp signal given by time-frequency is shown in Figure 1.

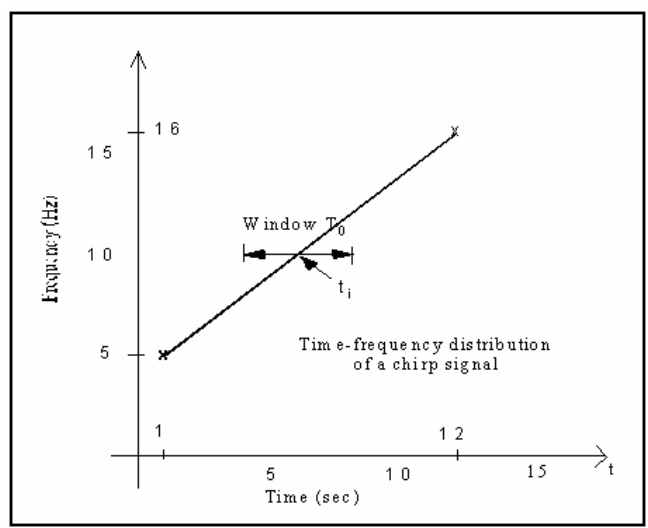

Figure 1 Chirp Signal in Time-Frequency Display.

Firstly, the two end points $t=1 \mathrm{~s}$ and $t=12 \mathrm{~s}$ respectively was considered. Accordingly, the representation of chirp signals will become purely sinusoidal of frequency $5 \mathrm{~Hz}$ and $16 \mathrm{~Hz}$ as depicted in Figure 2 and Figure 3 respectively.

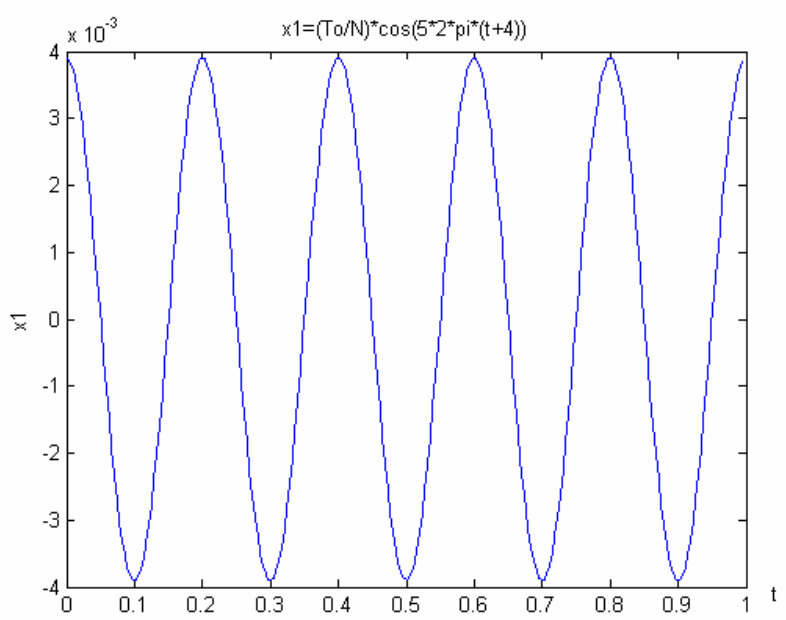

Figure 2 a 5 Hz Chirp Signal. 


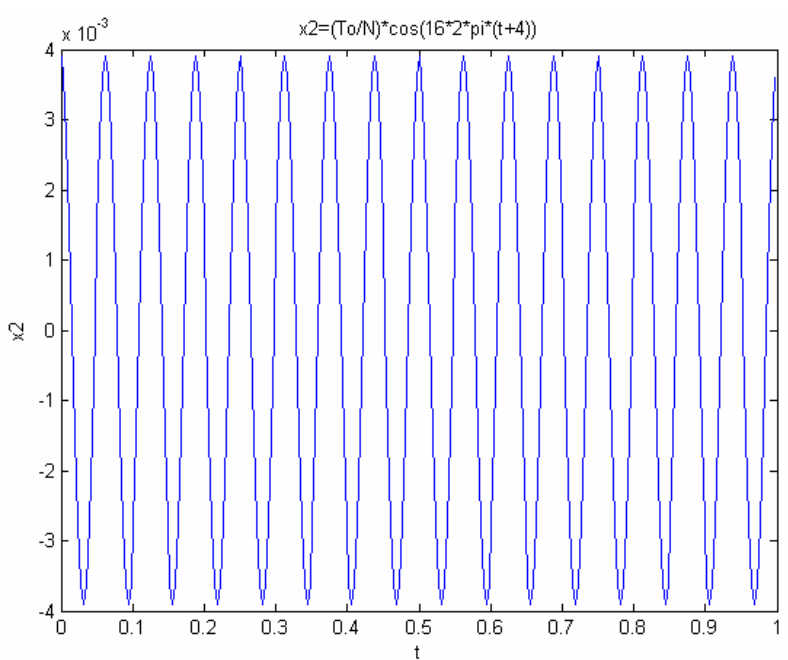

Figure 3 a 16 Hz Chirp Signal.

The value of window width $T_{o}$ and sampling time $T$ have been selected such that the number of $\mathrm{N}=256$ for each display. The resulting DFT experiment of each Chirp signal is obtained as follows:

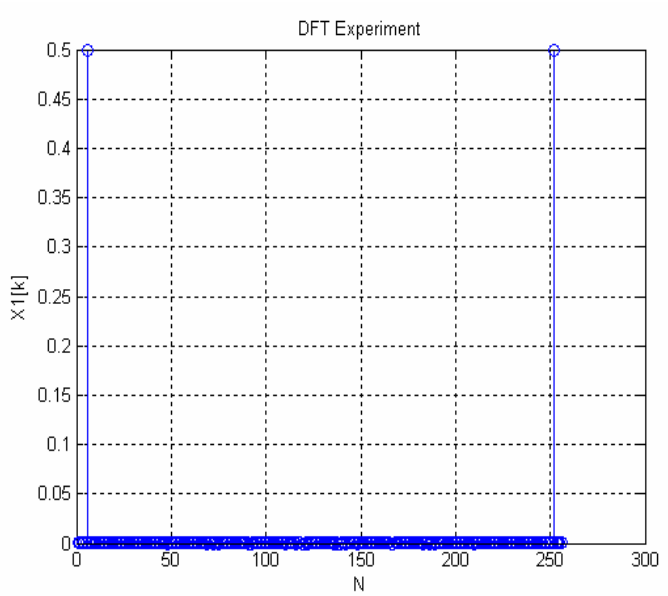

Figure 4 The Resulting DFT for $\mathrm{x}_{1}(\mathrm{t})$.

Since in Matlab, life begins with $\mathrm{n}=1$, the biggest $x(k)$ with amplitudes 0.5 are located on $\mathrm{N}=6$ and $\mathrm{N}=252$. The sum of squares $\sum\left|X[k] / T_{o}\right|^{2}$, as an average power representation has been calculated to be exactly half a watt. It turns out 
that the largest $X[k]$ components have been placed in the right frequency bin and of the right values.

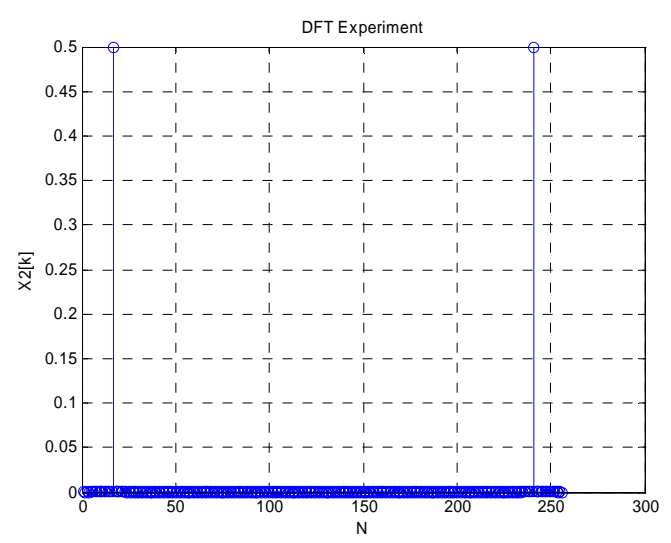

Figure 5 The Resulting DFT for $x_{2}(t)$.

Similarly, the same reason applied for allocating the biggest $x(k)$ with amplitude 0.5 on $\mathrm{N}=17$ and $\mathrm{N}=241$ which theoretically they should be on $\mathrm{N}=16$ as well as $\mathrm{N}=240$. Again, the sum of squares $\sum\left|X[k] / T_{o}\right|^{2}$, as an average power representation has been calculated to be exactly half a watt. Likewise, it turns out that the largest $X[k]$ components have been placed in the right frequency bin and of the right values.

\subsection{3-D Spectral Contents against Time $t_{i}$ and Frequency Bin $k$}

Now, a rectangular sliding window $w(t, \tau)$ is centred at a fixed time $t$, with a window width defined as: $x_{w}(t, \tau)=x(t+\tau) w(t, \tau)$ within $-T_{o} / 2<\tau \leq T_{o} / 2$, in which $t$ is called the frame time. It is fixed within a window time. Also, $\tau$ is regarded as a running variable, which varies from range $-T_{o} / 2$ to $T_{o} / 2$ for each window frame. A block of $N=T_{o} / T$ data was obtained, which $t_{i}=0,0.5,1,1.5, \ldots, 15$. Consequently, a block of 31 data, each of 256 values was examined. The next step is to present the spectral contents $\sum\left|X\left[t_{i}, k\right] / T_{o}\right|$ against time $t_{i}$ and frequency bin $k$.

As a result, we obtain the 3D plot time-frequency distribution as follows: 


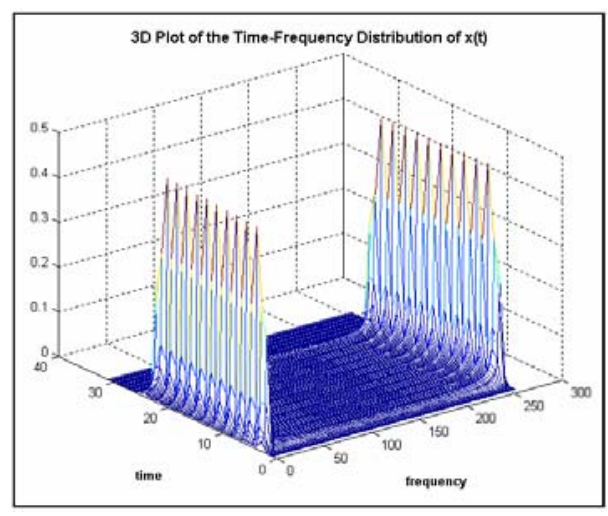

Figure 6 3-D Plot of Noiseless Time-Frequency Distribution of $x(t)$.

Due to the fact that the range of $t$ starts from 1 to 12 , some data have zeroes value in the beginning and in the end of simulation as shown in Figure 5 and Figure 6 . Subsequently, the total average power against various value of $t_{i}$ is depicted in Figure 7.

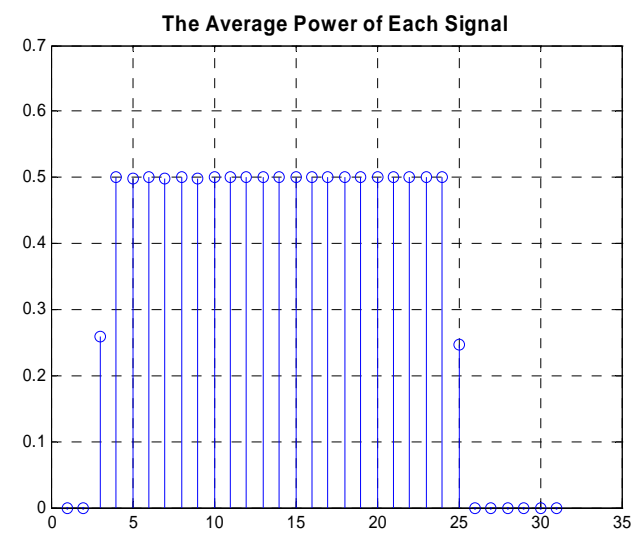

Figure 7 Total Average Power against Various Value of $t_{i}$.

Apparently, for $\mathrm{t}=1$ and $\mathrm{t}=2$, the average power equals to zero, due to the fact that no chirp signal appeared in the systems. The same condition applied for $\mathrm{t}=26$ up to $\mathrm{t}=31$. The point to remember is that index in Matlab always starts from $n=1$. 


\subsection{The Effect of Noise}

One watt of Gaussian noise is now added to the systems. As a result, the corrupted signals are depicted in Figure 8.

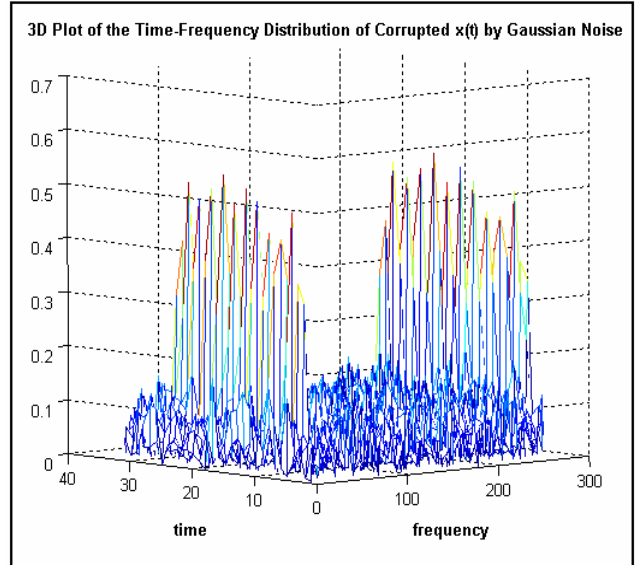

Figure 8 Signals Corrupted by One Watt Gaussian Noise.

From the time-frequency plot in Figure 8 one can still be possible to visually discern the presence of the chirp signals amongst the noises. This, of course, will make a significant impact on the average power of the signals, as shown in Figure 9.

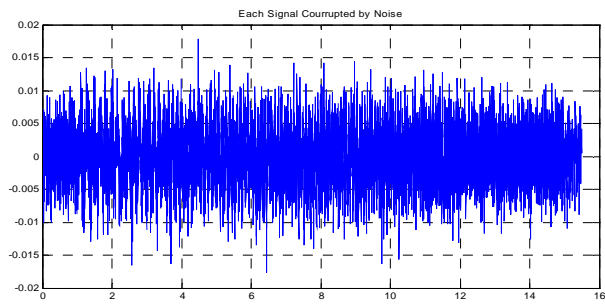

Figure 9 Noise in Time Domain Representation.

Accordingly, the effects of Gaussian noise on the average power are depicted in Figure 10. 


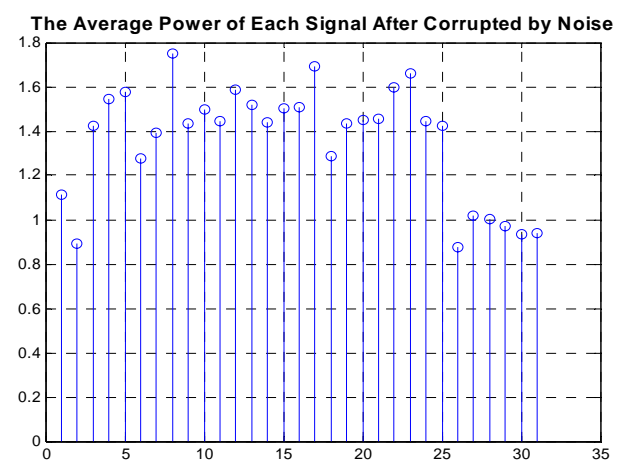

Figure 10 The Effect of Noise on The Average Power.

As can be seen, the average power of the signals due to one watt Gaussian noise is no longer half watt. They have fluctuated between about one and one and half watt. What is more, the zero values power of signals is no longer appear. Roughly speaking, at that time distribution the average power of signals have become around one watt. It corresponds to the one watt Gaussian noise. (see $\mathrm{t}=1,2,26-31)$.

\subsection{De-noising Techniques Using P-Welch Function}

The underlying principles of Welch based denoising technique can be rigorously discussed as follows [10]:

Firstly, the input signal vector $x$ is broken into $k$ overlapping segments according to window and $n$ overlap (or their default values). If the window size is larger than the number of FFT points (NFFT), the signal is divided into NFFT-length segments and then, the last segment is padded with zeros. Subsequently, the specified (or default) window is applied to each segment of $x$. (No preprocessing is done before applying the window to each segment.).

Furthermore, an $\mathrm{n}$ fft-point FFT is applied to the windowed data and the (modified) periodogram of each windowed segment is computed. The set of modified periodograms is averaged to form the spectrum estimate $S\left(e^{\mathrm{j} \omega}\right)$.

The resulting spectrum estimate is scaled to compute the power spectral density $S\left(e^{j \omega}\right) / F$, where $F$ is

- $2 \pi$ when we do not supply the sampling frequency

- fs when we supply the sampling frequency 
The performances of Welch based de-noising techniques with respect to power spectral density function are now examined. We shall also investigate the threshold of noise power in which the additional noise can collapse the chirp signals. Computer simulations have been started from the level of 1 watt, 1.5 watt up to 3.5 watt noise. A More comprehensive discussion will be presented accordingly.

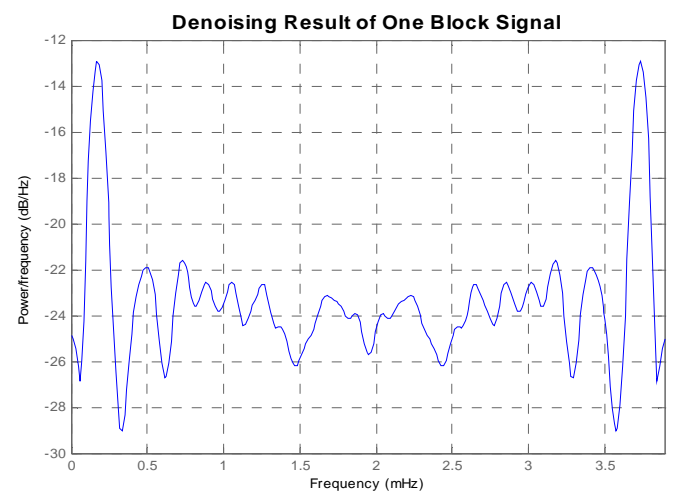

Figure 11 2D Representation of De-Noising Signals (2D).

Figure 11 and Figure 12 depict the result of the P-Welch de-noising process. As indicated, one can still discern the presence of the chirp signals amongst the noises. The noise power applied is one watt Gaussian noise.

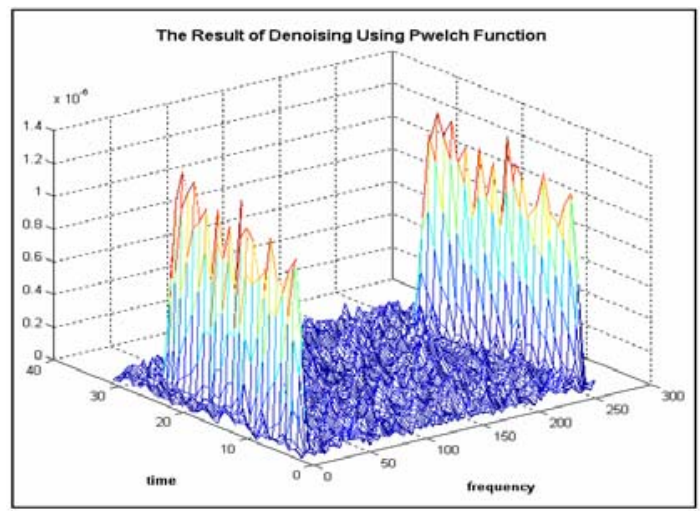

Figure 12 3D Representation of De-Noising Signals.

Furthermore, we now set the noise power into 1.5 watt. As a result, the chirp signals are buried deeper under Gaussian noise. One still may be able to discern it. Nevertheless, it has been progressively more difficult to discern the chirp signals due to these circumstances. 


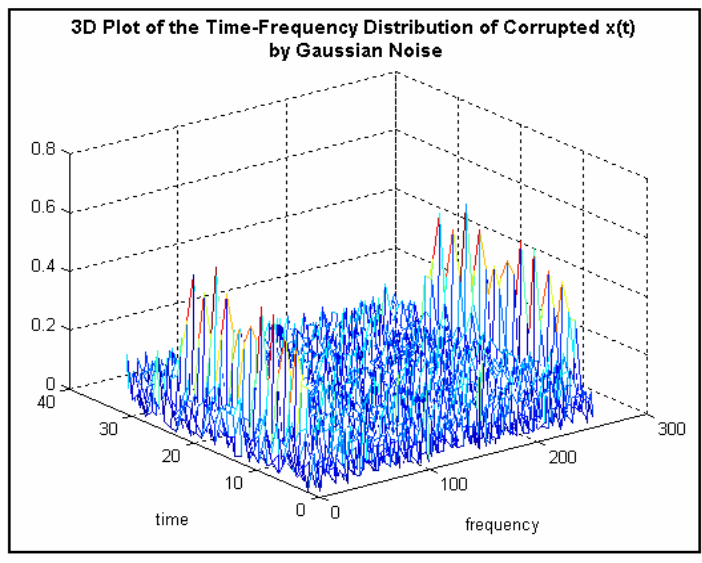

Figure 13 Signals Corrupted by 1.5 watt Gaussian Noise.

Upon this de-noising process, some of the noises have been successfully filtered out, and the presence of the chirp signals is more apparent as illustrated by Figure 14.

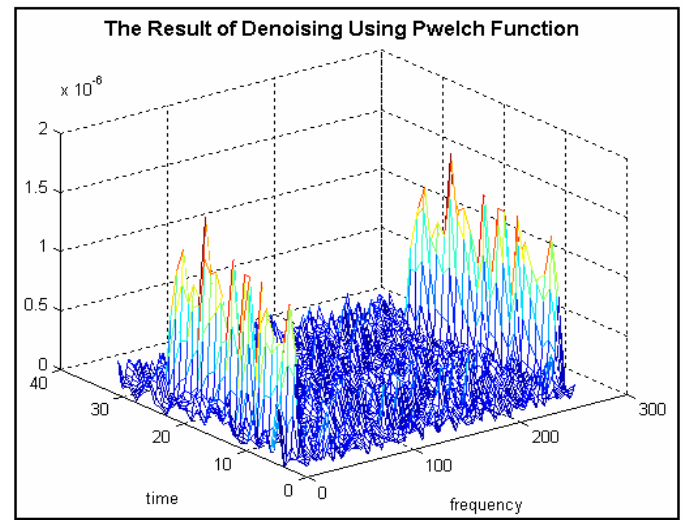

Figure 14 Representation of De-Noising Signals.

Subsequently, we now enhance the Gaussian noise power from 1.5 watt into 2 watt. Accordingly, the signals are mostly collapsed due to the increasing noise power as shown in Figure15. 


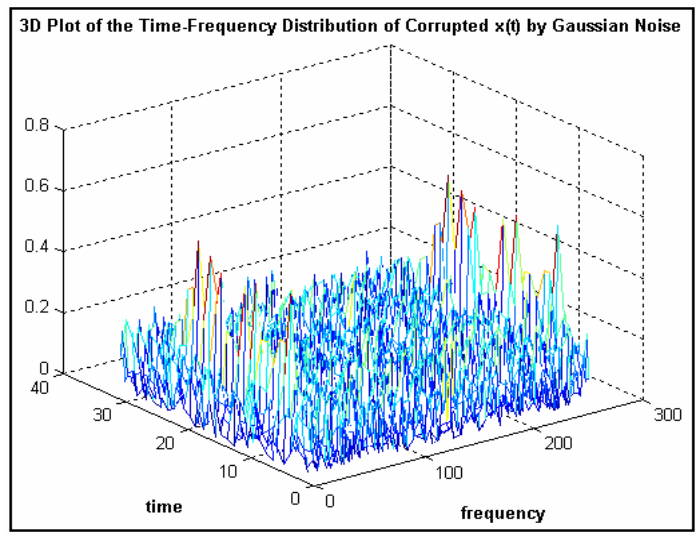

Figure 15 The Effect of 2Watt Gaussian Noise.

Upon performing P-Welch de-noising technique, the signals to noise ratio due to 2 watt Gaussian noise has been significantly improved.

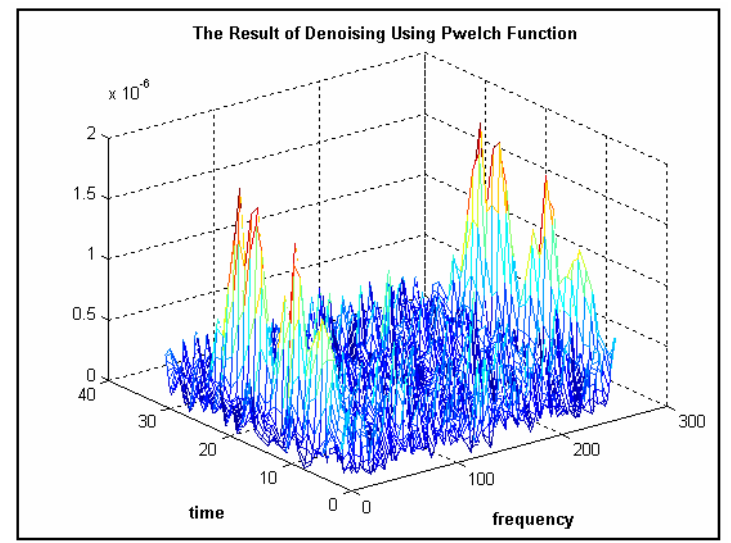

Figure 16 Representation of De-Noising Signals.

Nonetheless, as the noise power has significantly increased, the resulting denoising signals in terms of $\mathrm{S} / \mathrm{N}$ ratio have decreased moderately.

The question we are attempting to address now is what the unrecoverable threshold of Gaussian noise power. To address this question, again, we increase the noise power into 2.5 watt. 


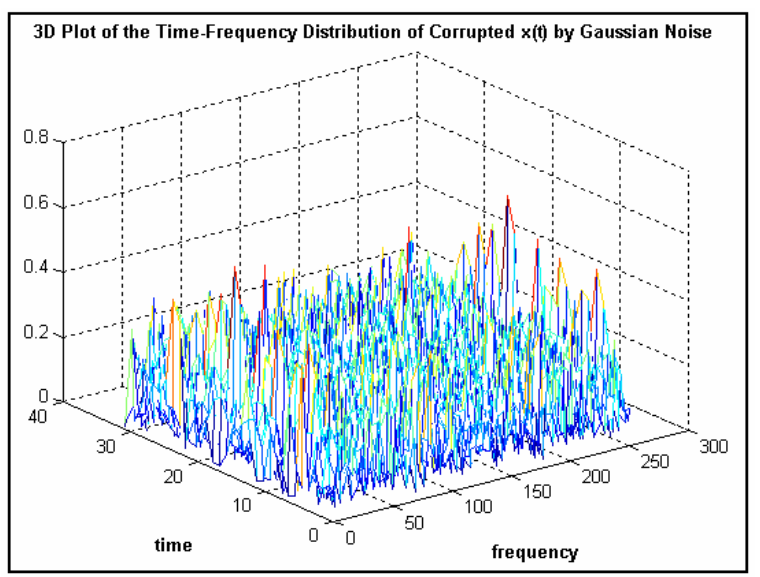

Figure 17 The Effect of 2.5 Watt Gaussian Noise.

From Figure 17, it is apparent that the chirp signals have been totally buried under the noise.

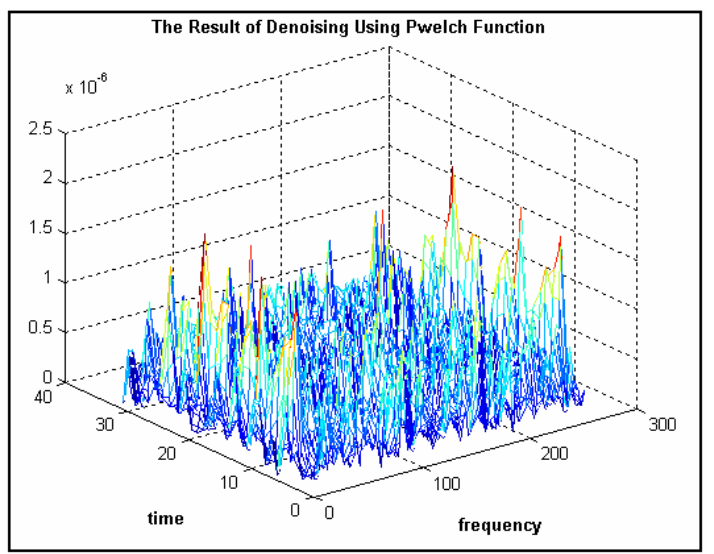

Figure 18 Representations of De-Noising Signals 3 Watt Gaussian Noise.

According to Figure 18, P-Welch de-noising process does not mean much to recover the original signal. The signals can be substantially recovered, albeit the $\mathrm{S} / \mathrm{N}$ ratio is still poor.

Moreover, 3 and 3.5 watt additional Gaussian noise will result in the chirp signals given by Figure 19 and Figure 20. 


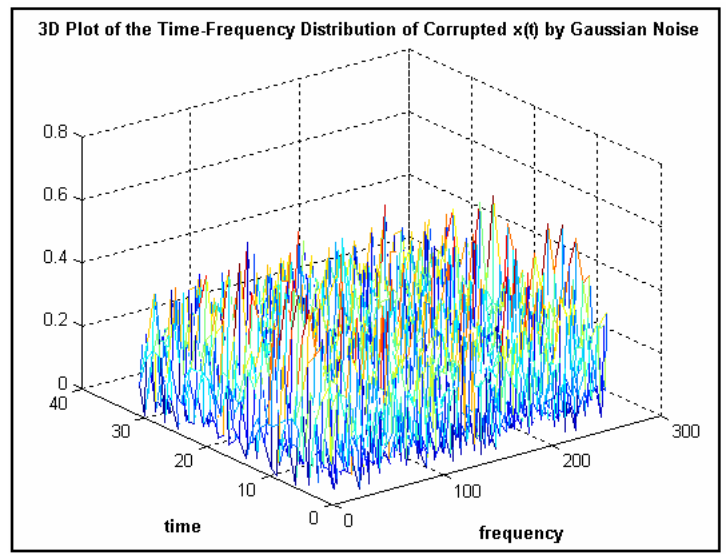

Figure 19 The Effect of 3 Watt Gaussian Noise.

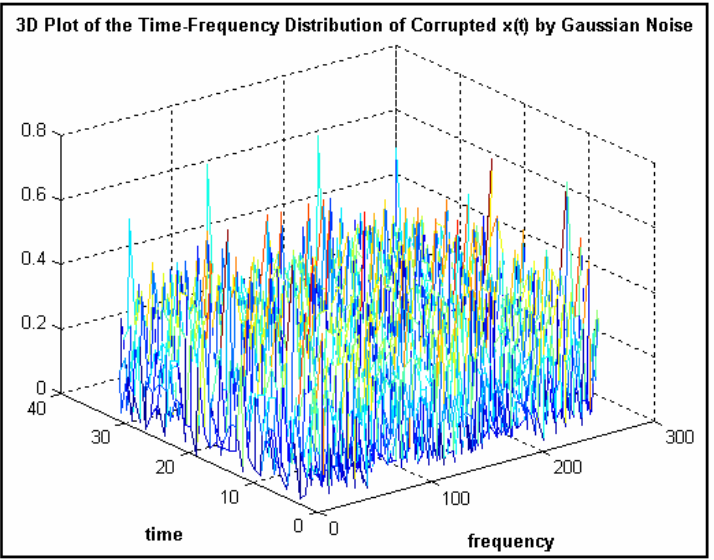

Figure 20 The Effect of 3.5 Watt Gaussian Noise.

As the ratio of $\mathrm{S} / \mathrm{N}$ is poorer, it is now completely impossible to visually discern the original signals and the noise.

Upon performing P-Welch Denoising technique, we still end up with poor signal to noise ratio as indicated by Figure 21 and Figure 22. 


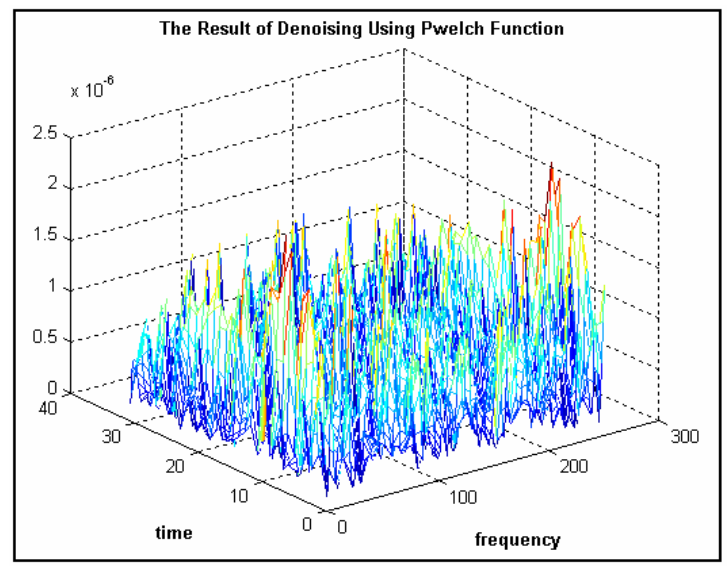

Figure 21 De-Noising Signals due to 3Watt Gaussian Noise.

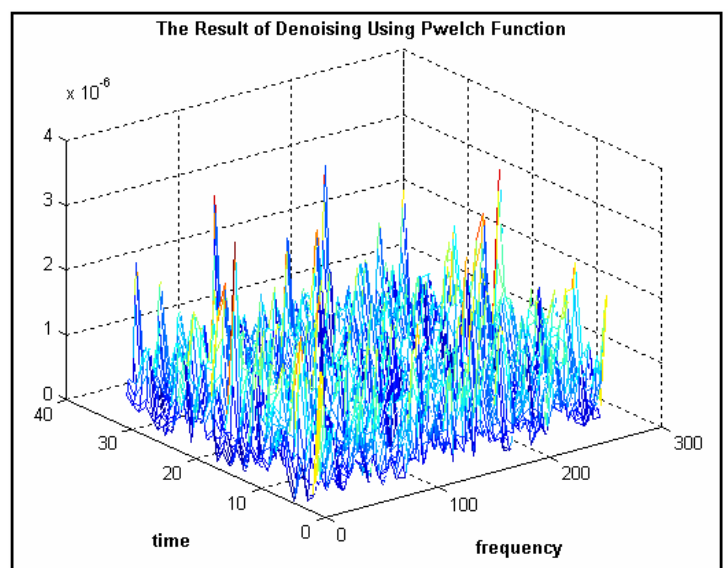

Figure 22 De-Noising Signals due to 3.5 watt Noise.

It can be concluded that although the de-noising process has been performed the desired signal and the interference are not discernable should the noise power has exceeded its threshold value. In this study, we have a threshold noise power about 2.5 watts.

\section{$4 \quad$ Concluding Remarks}

To sum up, without employing de-noising techniques, the presence of chirp signals that still can be visually discerned is up to 2 watt Gaussian noise power. However, since the power of the noise has gradually increased, exceeding a threshold value, say, above 2 watts (see Fig 17, 19 and 20) the chirp signals have been completely overwhelmed. By employing Pwelch de-noising 
technique, the desired signal still can be discernable in the presence of 2.5 or 3 watt Gaussian noise. Beyond this limit, we will end up with the unsatisfactory de-noising results, as indicated by poor $\mathrm{S} / \mathrm{N}$ ratio results. For comparison, radar can only detect the presence of the aircraft at a certain limited distance only. If the aircraft moves further apart, then the transmitted signals become weaker and weaker before the noise can completely overwhelm it. It turns out that the presence of the aircraft is no longer perceptible.

\section{Acknowledgement}

The author wishes to thank Dr. Khee K. Pang, a reader at the Department of Electrical and Computer Systems Engineering, Monash University, Australia for the completion of this research project.

\section{List of References}

[1] Keogh D. B. and Pang K. K., Signal Processing, Mi-tec, Monash University, 2005.

[2] Oppenheim, A.V. \& Schafer, R.W., Digital signal processing, PrenticeHall 1989.

[3] Proakis, J. G., Salehi M., and Bauch G., Contemporary Communication Systems, Thomson Brooks/Cole, Canada, 2004.

[4] The Use of Fast Fourier Transform for the Estimation of Power Spectra: A Method Based on Time Averaging Over Short, Modified Periodograms, IEEE Transactions on Audio Electro-Acoustics, Volume AU-15 (June 1967), pages 70-73.

[5] Oppenheim, A.V., and R.W. Schafer, Digital Signal Processing, Englewood Cliffs, NJ: Prentice-Hall, 1975, pp 548-554.

[6] Proakis J., Salehi M., Communication Systems Engineering, Prentice Hall, New Jersey, 2002.

[7] Proakis, J., Digital Communications, McGraw-Hill International Edition, Singapore, 2001.

[8] Jayant N.S. and Noll P., Digital Coding of Waveforms - Principles and Applications to Speech and Video, Prentice-Hall 1984.

[9] Premkumar B., Principles of Wireless Communications, Pearson Prentice Hall, Singapore, 2004.

[10] Signal Processing Toolbox, www.mathworks.com. 